\title{
MOLECULAR RECOGNITION IN GAS SENSING: RESULTS FROM ACOUSTIC WAVE AND IN-SITU FTIR MEASUREMENTS
}

\author{
Andreas Hierlemann and Antonio J. Ricco \\ Microsensor Research \& Development Department, Sandia National Laboratories \\ Albuquerque, New Mexico 87185-1425 \\ Karl Bodenhöfer and Wolfgang Göpel \\ Institut für Physikalische und Theoretische Chemie, Universität Tübingen, Auf der Morgenstelle 8 \\ 72076 Tübingen, Germany
}

\begin{abstract}
Surface acoustic wave (SAW) measurements were combined with direct, in-situ molecular spectroscopy to understand the interactions of surface-confined sensing films with gas-phase analytes. This was accomplished by collecting Fourier-transform infrared external-reflectance spectra (FTIR-ERS) on operating SAW devices during dosing of their specifically coated surfaces with key analytes.
\end{abstract}

\section{INTRODUCTION}

Molecular recognition is an important topic when searching for new, selective coating materials for chemical sensing. Recently, the general idea of molecular recognition in the gas phase was challenged by Grate et al. [1]. However, in earlier thicknessshear mode resonator (TSMR) investigations, convincing evidence was presented for specific recognition of particular analyte target molecules $[2,3]$.

In this study, we systematically investigated coatings previously shown to be highly selective, such as the bucket-like cyclodextrins for chiral recognition [2], Ni-camphorates for the specific detection of the bases pyridine [3] and DMMP (dimethylmethylphosphonate), and phthalocyanines to specifically detect benzene, toluene, and xylene (BTX). In the case of the cyclodextrin (CD), oriented adsorption of the molecules within the $\mathrm{CD}$ torus (inclusion of the analyte molecule in the supramolecular cage) seems to control the recognition process; in the case of $\mathrm{Ni}$ camphorate, it is probably coordinative ligand-metal donor bonding; for the phthalocyanines, it is likely " $\pi$-stacking", an intense interaction of the delocalized $\pi$ electron systems of the phthalocyanine and the volatile aromatic compounds.

To further elucidate these interactions, we performed combined SAW/in-situ FTIR-ERS measurements. During each experiment, the film-coated SAW device, which simultaneously acts as a reflector for an IR beam at grazing incidence, is exposed to various volatile organics. From simultaneously recorded changes in the device frequency (principally due to mass changes) and changes in the IR signature of the volatile organic compound, one can reach fundamental conclusions about the coating/analyte interaction processes. Suitable control experiments, using "ordinary" organic polymers expected to show only simple physical interactions with the analytes, were conducted for comparison.

\section{EXPERIMENTAL DETAILS}

The coating materials chosen for this study (Fig. 1) were: a modified $\gamma$-cyclodextrin (3-O-butanoyl-2,6-di-O-n-pentyl- $\gamma$-cyclodextrin, CD) as cage compound; a nickel camphorate (Ni(II)bis[(1R)-(+)-3-heptafluorobutanoyl-8-methylidencamphorate, Nicam) as coordinating agent; and a soluble nickel phthalocyanine (Ni-oktakis-O-pentyl-phthalocyanine, $\mathrm{Ni}-\mathrm{PC}$ ) as a material with a large, delocalized $\pi$-electron system. In addition, we used the reference polymers poly(etherurethane) (PEUT; Thermedics Inc., Woburn, MA) [2] and polyisobutylene (PIB, $M W \approx 380,000$; Aldrich, Milwaukee, WI). The coatings on the SAW/FTIR-devices were prepared by spin casting or spray coating from methylene chloride solutions.

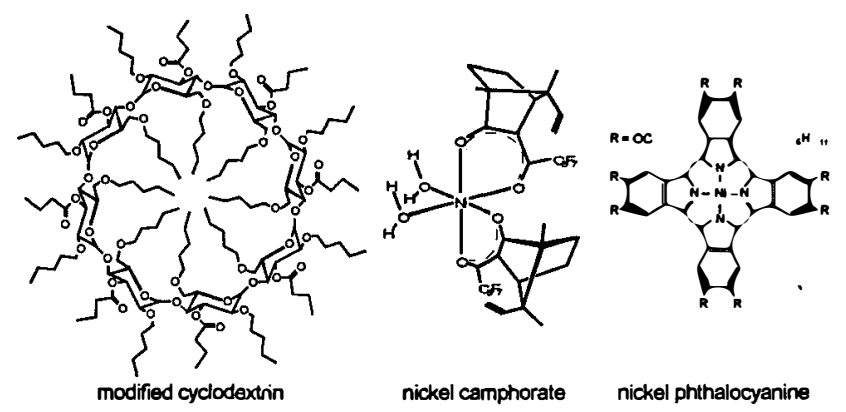

Figure 1. Molecular recognition coating materials used in this study.

The TSMR array consisted of discrete piezoelectric quartz crystals (AT-cut) with gold electrodes operating at a fundamental frequency of $30 \mathrm{MHz}$ (plate thickness: $55.6 \mu \mathrm{m}$ ) purchased from Kristallverarbeitung Neckarbischofsheim, Germany [4].

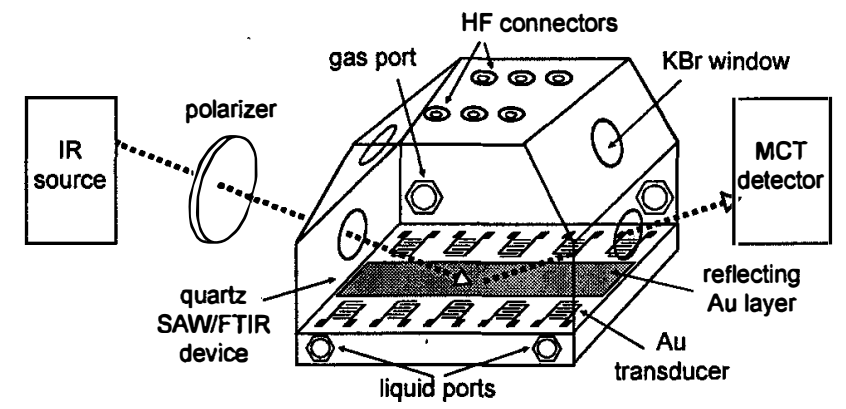

Figure 2. Setup for simultaneous SAW/in-situ FTIR external reflectance measurements.

SAW devices were custom designed at Sandia National Laboratories to provide a large gold reflection area for the FTIR measurements (Fig. 2). Five sets of independent interdigital $\mathrm{Au}$ transducers were defined on a $30 \times 10 \mathrm{~mm} \mathrm{ST-quartz}$ substrate; only the three center delay lines were used for measurements. The operating frequency of the devices was $97 \mathrm{MHz}$. SAW measurements were performed according to previously described procedures [5]. The SAW/FTIR devices were mounted in a custom gasflow test cell (Fig. 2), which was attached to a gas manifold, using nitrogen as the carrier gas and bubblers for vaporizing the volatile organics.

FTIR measurements were performed using a Nicolet MagnaIR 750 spectrometer. Reflection spectra were obtained using a Harrick Scientific (Ossining, NY) Seagull accessory with an inci- 
dent angle of $80^{\circ}$ off normal. The orientation of the IR radiation ( $s$ or $p$ ) was selected by a wire-grid polarizer.

Volatile organics were used as received from Aldrich, Milwaukee, WI; the pure enantiomeric compounds were purchased from Fluka, Buchs, Switzerland.

\section{RESULTS AND DISCUSSION}

Our SAW/FTIR results support earlier conclusions derived from TSMR data $[2,3]$. In most cases where specific chemical interactions-metal coordination, cage compound formation, or pi-stacking - are expected, we record distinctive changes in the IR spectra, together with anomalously large SAW sensor responses, upon analyte dosing. In contrast, for standard physisorption processes (i.e., the "standard" polymers PEUT or PIB with various volatile organics), no changes in the IR spectra occur and the SAW responses are much smaller. In addition, the partition coefficients (or SAW sensor signals) roughly followed the fraction of saturation vapor pressure (variations do occur when comparing the nonpolar PIB to the polar PEUT).

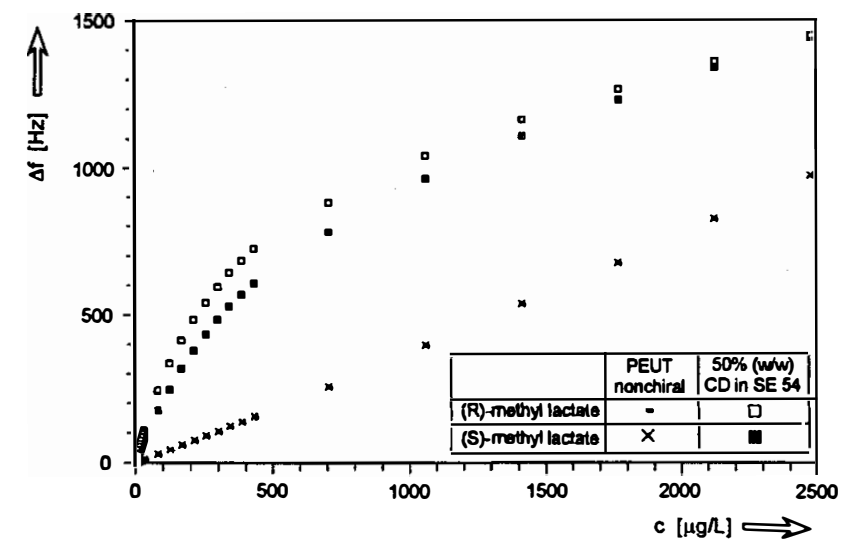

Figure 3. Typical sensor responses representing preferential sorption (bent curves) and standard physisorption (straight line).

Fig. 3 shows the response of a CD-coated TSMR upon exposure to methyl lactate over a wide concentration range. A curve typical of preferential sorption is shown: Langmuir-type behavior in the low concentration range gives way gradually to a linear physisorption curve, similar to that depicted for PEUT. Similar nonlinear sorption behavior at low analyte concentrations occurs for any of the investigated "molecular recognition" coatings with the corresponding specifically detectable molecules, including $C D$ with methyl lactate; Ni-camphorate with pyridine or DMMP; and Ni-PC with aromatics like the BTX compounds.

Fig. 4 shows portions of the IR-spectra of methyl lactate recorded simultaneously with the SAW response. There are three main changes in the spectrum of methyl lactate sorbed into the $C D$ in comparison to the reference spectra of the same compound in PEUT or PIB: (1) a new peak in the $\mathrm{OH}$ region at $3470 \mathrm{~cm}^{-1}$; (2) a shoulder in the $\mathrm{CO}$ region at $1725 \mathrm{~cm}^{-1}$; and (3) a new peak at $1190 \mathrm{~cm}^{-1}$. The intensity of all these peaks for $s$-polarized light (not shown) is considerably lower than for the $p$-polarized light (Fig. 4), the latter having greater sensitivity to near-surface dipoles [6]. The intensity also varies somewhat between the $R$ and $S$ enantiomers, the changes in the $R$-methyl lactate spectrum being more pronounced than those in the spectrum of the $S$ enantiomer, consistent with stronger sorption of the $R$ enantiomer into the CD matrix as previously shown by GC and TSMR measurements [3].

\section{CONCLUSIONS}

The method of simultaneous SAW/in-situ FTIR measurements is an effective, powerful tool for elucidating interactions between coatings and gaseous analytes, providing direct evidence for molecular recognition in acoustic wave gas sensors. Sandia is a multiprogram laboratory operated by Sandia Corporation, a Lockheed Martin Company, for the United States Department of Energy under Contract DE-AC04-94AL85000.

\section{REFERENCES}

1. J. W. Grate, S. J. Patrash, and M. Abraham, "Selective Vapor Sorption by Polymers and Cavitands on Acoustic Wave Sensors: Is This Molecular Recognition?", Anal. Chem., 68, 913 (1996).

2. A. Hierlemann, K. Bodenhöfer, M. Fluck, V. Schurig, and W. Göpel, "Selective detection of Nitrogen and Oxygen Containing Volatile Organic Compounds...", Anal. Chim. Acta, 346, 327 (1997).

3. K. Bodenhöfer, A. Hierlemann, M. Juza, V. Schurig, W. Göpel, "Chiral Discrimination of Inhalation Anesthetics and Methyl Propionates by Thickness Shear Mode Resonators...", Anal. Chem., 69, 4017 (1997).

4. K. Bodenhöfer, A. Hierlemann, G. Noetzel, U. Weimar, and W. Göpel, "Performance of Mass-Sensitive Devices for Gas Sensing: Thickness Shear Mode and Surface Acoustic Wave Transducers", Anal. Chem., 68, 2210 (1996).

5. R. C. Thomas, H. C. Yang, C. R. DiRubio, A. J. Ricco, and R. M. Crooks, "Chemically Sensitive Surface Acoustic Wave Devices...", Langmuir, 12, 726 (1996).

6. R. M. Crooks, L. Sun, C. Xu, S. L. Hill, and A. J. Ricco, "The Characterization of Organic Monolayers by FTIR-ERS", Spectroscopy, 8, 28 (1993).
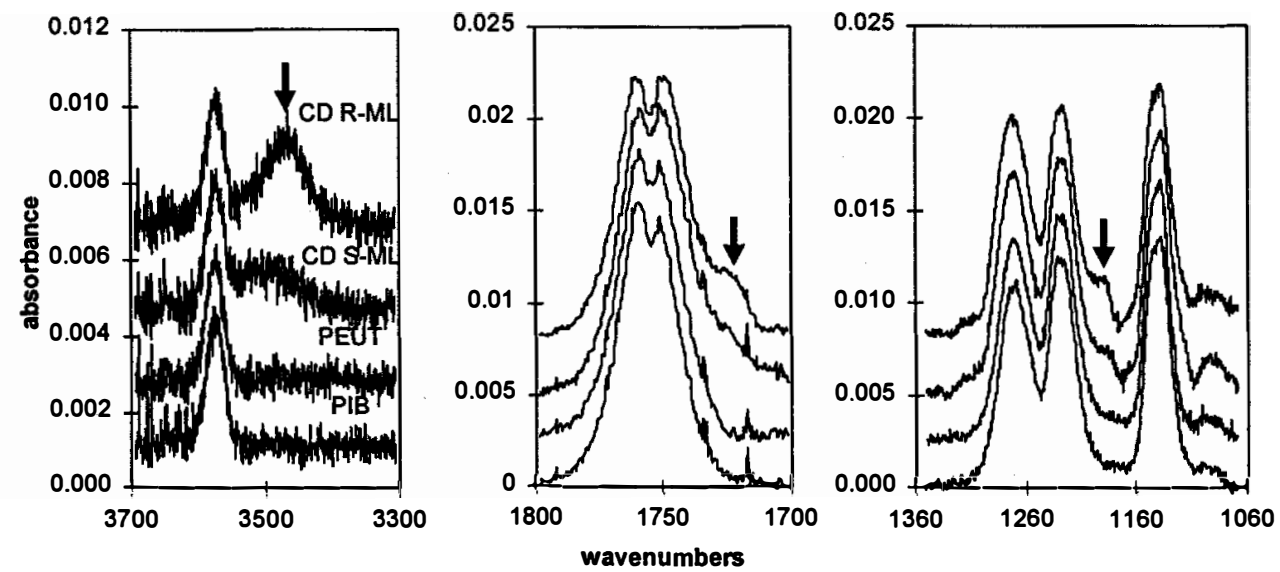

Figure 4. IR reflectance spectra of both enantiomers of methyl lactate (R-ML and S-ML) on CD and the polymers PIB and PEUT. 